\title{
PACKET DESIGN FOR COMMUNICATION UNDER ASYNCHRONOUS INTERFERENCE
}

\author{
Cristian Budianu and Lang Tong \\ School of Electrical and Computer Engineering, \\ Cornell University, Ithaca, NY, USA \\ \{cris, ltong\}@ece.cornell.edu
}

\begin{abstract}
The asynchronous interference arises in many wireless multiple access communication systems. This paper considers the problem of placing the training symbols within the data packets when these packets are subject to asynchronous interference. The performance measure considered is the achievable data transmission rate for a system that uses coherent reception using a training-based channel estimate. An upper and a lower bound for the achievable rate are obtained. By optimizing the placement the achievable rate can be doubled. Also, if the the placement that provides optimum channel estimation performance is used, the data rate loss is negligible; thus the effect of the placement on the data rate is mainly through the efficiency of channel estimation.
\end{abstract}

\section{INTRODUCTION}

Asynchronous interference arises in ad-hoc networks, wireless LANs, and even in cellular networks in the form of co-channel interference. In packet wireless networks with a large number of users, for example, it is hard to schedule the packets of different users to avoid collisions; sometimes it is not possible that these packets are synchronized. Moreover, the interference packets can have a large range of power levels due to the near-far effect.

This paper investigates the influence of the packet design on the achievable data rates in such a scenario. The main assumption is that the channel is unknown to both transmitter and receiver and the receiver uses an estimate of channel state information (CSI) to recover the data symbols.

To acquire CSI, training symbols are often inserted in the data stream in a predetermined pattern. Once the power allocated to the training symbols is fixed, the channel estimation performance depends on the placement of the training symbols. Although the channel estimation performance affects the achievable rates, usually the placements that optimize the achievable rate and the channel estimation perfor-

This work was supported in part by the Multidisciplinary University Research Initiative (MURI) under the Office of Naval Research Contract N00014-00-1-0564, and Army Research Laboratory CTA on Communication and Networks under Grant DAAD19-01-2-0011. mance are not the same. Placement optimization has been considered in [1-5] in different frameworks, and using different metrics.

In the system considered the data is transmitted in packets and the channel is considered Rayleigh block-flat-fading, constant for each packet and independent from packet to packet. The asynchronous interference is modeled as one packet of unknown symbols that overlaps partially with the data packet; the relative position of these two packets is random and distributed uniformly, so that the average number of data and training symbols "hit" by the interference is the same for all placements. However, the placement determines the distribution of the number of data and training symbols that are hit; this distribution affects the MMSE estimator, its MMSE, and the achievable rate. For example, if all training symbols are placed in one cluster, then it is likely that either all training symbols get hit or none of them, while if the training symbols are spread uniformly in the packet, any number of training symbols can be hit equally likely.

Since the receiver does first estimation and then decoding, we first describe the channel estimator considered in the analysis. The optimal MMSE estimator is non-linear and thus is analytically hard to use. One way to overcome this difficulty is to consider a lower bound given by the MSE of a linear estimator that knows the position of the interference with respect to the data packet. This MSE is the genie (lower) bound and its dependence on the placement is determined completely and the placement that minimizes the genie bound is in two clusters of equal or quasi-equal length at the two edges of the data packet. In [6] it is shown that the genie bound is tight when the interference power is high, which means that in this case the placement mentioned before minimizes the MMSE.

Considering a receiver that uses the genie estimator, we determine achievable rates of a system that uses BPSK signaling in two scenarios - hard decoding and soft decoding. Evaluating numerically these expressions it is shown that the effect of the placement on the achievable rates is mainly through channel estimation. In other words, the gain obtained by using the optimal training scheme over the training scheme optimized for channel estimation performance is negligible. 
Existing work on optimal placement focuses mainly on channels where self interference is introduced by channel memory $[2,3]$. In such cases and under different metrics, the optimal placements tend to be scattered. In particular, for ISI channels, the optimal placement of training symbols is the quasi-periodic placement [2] where the pilot symbols are placed periodically with the minimum cluster size. When the interference comes from asynchronous packets, this result holds partially, but the pilot symbols are not distributed uniformly anymore, and tend to be concentrated toward the edges of the data packet. Moreover, the placement that optimizes the achievable data rate depends on the SNR of the system. However, using the optimal placement strategy for channel estimation where two training clusters are placed at the two ends of the packet provides near-optimum performance.

Notations : the vectors are in bold fonts, $\mathbb{E}_{X}$ is the expectation with respect to the random variable $X, \mathbb{P}\{A\}$ the probability of the event $A, \operatorname{diag}(\mathbf{A})$ is a column vector formed by the diagonal elements of the square matrix A. We use $\mathbf{1}_{u}$ to denote an $u \times 1$ vector with all elements equal to 1 and $\mathbf{1} \triangleq \mathbf{1}_{N}$. Sometimes the same function $f(\cdot)$ is written $f(\cdot ; a)$ to emphasize the dependence on the parameter $a$. Given the vector $\mathbf{a}, a_{k}$ is its $k$-th element. The Complex Gaussian distribution with mean $\boldsymbol{\mu}$ and covariance matrix $\mathbf{C}$ is denoted by $\mathcal{C N}(\boldsymbol{\mu}, \mathbf{C})$.

\section{SYSTEM MODEL AND PROBLEM FORMULATION}

Consider a point-to-point one-way communication link. The data is transmitted in packets of $B$ symbols, and the channel is assumed Rayleigh block-flat-fading, constant during the transmission of one packet and independent from packet to packet. The received signal is given by the $B \times 1$ vector

$$
\mathbf{y}=\mathbf{s} A+\mathbf{z} .
$$

In the equation above $A \sim \mathcal{C N}(0,1)$ is the complex scalar channel parameter, s a $B \times 1$ vector representing one block of transmitted symbols and $\mathbf{z}$ the total noise vector; its probability density function (pdf) will be derived. We assumed the variance of the channel equal to 1 without loss of generality.

The total noise vector $\mathbf{z}$ is the sum of two independent random vectors - the usual i.i.d. complex additive white Gaussian noise (CAWGN) with zero mean and known variance $\sigma^{2}$, and the asynchronous interference. The interference which is modeled as a packet with the same length $B$ as the data packet, having i.i.d CAWGN symbols with zero mean and known variance $\sigma_{q}^{2}$. The interference and data packets overlap in a random position $V$ distributed uniformly on $\{1, \ldots, 2 B-1\}$ (Fig. 1). If $V \in\{1, \ldots, B\}$ then the first $V$ symbols of the data packet are hit and if $V \in\{B+1, \ldots, 2 B-1\}$ then the last $2 B-V$ symbols are hit. Note that we assumed that the data and interference packets always overlap in at least one position. The position of the interference is unknown to the transmitter and receiver.

\section{CHANNEL ESTIMATION}

The data packet has $N \geq 2$ training symbols. Denote by $\mathcal{J}=\left\{t_{1}, \ldots, t_{N}\right\} \subset\{1, \ldots, B\}, 1 \leq t_{1}<t_{2}<\cdots<$ $t_{N} \leq B$, the ordered set of indexes of the training symbols within the packet. Extracting these symbols into an $N \times 1$ vector $\mathrm{x}$ we have

$$
\mathbf{x}=\mathbf{P y}=1 A+\mathbf{P z}=1 A+\mathbf{n} .
$$

The placement matrix $\mathbf{P}$ is an $N \times B$ matrix, with elements $\mathbf{P}\left(k, t_{k}\right)=1, \forall k \in\{1, \ldots, N\}$ and the rest of the elements 0 . We also supposed that all the training symbols are equal to 1 .

To do the channel estimation we need the distribution of the total noise and interference vector $\mathbf{n}$ that affects the training symbols. For this, we introduce another random variable, $U \in\{0,1, \ldots, 2 N-1\}$ that gives the position of the interference packet with respect to the training symbols (Fig. 1). Similar to $V$, if $U \in\{1, \ldots, N\}$ the first $N$ training symbols are hit and if $U \in\{N+1, \ldots, 2 N-1\}$ the last $2 N-U$ training symbols are hit. If $U=0$ then no training symbol is hit by the interference. $U$ is not distributed uniformly anymore; its distribution depends on the placement set $\mathcal{J}$. Defining $\varepsilon \triangleq \frac{1}{2 B-1}$ and the sets $\mathcal{S}_{N-1} \triangleq$ $\{1, \ldots, N-1\}, \mathcal{S}_{N+1} \triangleq\{N+1, \ldots, 2 N-1\}$, the distribution $p_{u} \triangleq \mathbb{P}\{U=u\}$ of $U$ is given by

$$
p_{u}=\left\{\begin{array}{lll}
\varepsilon\left(t_{u+1}-t_{u}\right) & \text { if } & u \in \mathcal{S}_{N-1} \\
\varepsilon\left(B-\left(t_{N}-t_{1}\right)\right) & \text { if } & u=N \\
p_{u-N} & \text { if } & u \in \mathcal{S}_{N+1} \\
p_{N}-\varepsilon & \text { if } & u=0
\end{array} .\right.
$$

We'll use $\mathbf{p} \triangleq\left[p_{0}, p_{1}, \ldots, p_{2 N-1}\right]^{T}$ to refer to the distribution of $U$. Also, denote by $\mathcal{P}$ the set of all distributions $\mathbf{p}$ that satisfy the conditions (3). Given a placement $\mathcal{J}$ and its distribution $\mathbf{p}$, define its mirror reflection by $\mathcal{J}^{\leftarrow} \triangleq\left\{B+1-t_{N}, \ldots, B+1-t_{1}\right\}$ and the corresponding $\mathbf{p}^{\leftarrow}$. Note that if the placement is symmetric we have $\mathbf{p}=\mathbf{p}^{\leftarrow}$. Because of the left-right symmetry of the model, the mirror reflection pairs behave identically.

Conditioned on the position of the interference $U=u$, the total noise $\mathbf{n}$ is Gaussian with independent elements, $\mathbf{n} \mid U=u \sim \mathcal{C N}\left(0, \mathbf{D}_{u}\right)$. For any $u \in\{0,1, \ldots, 2 N-1\}$ 


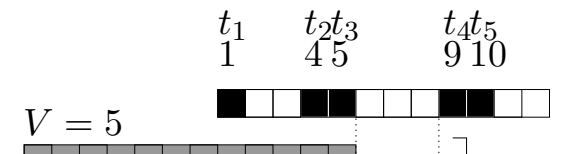

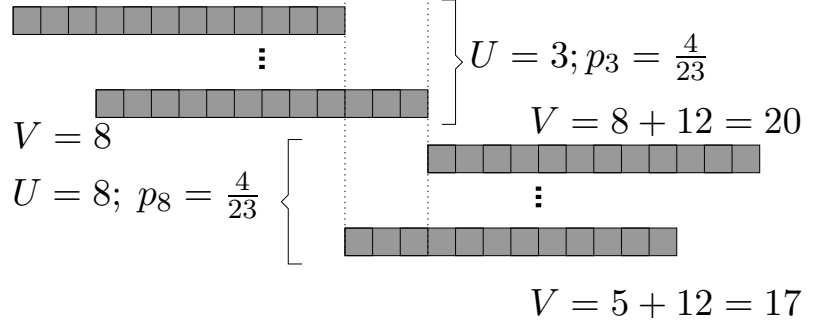

Fig. 1: Data packet and different possible positions of the interference packet. The training symbols are shaded in black. The interference packets are colored in gray. $B=12, N=5$, $\mathcal{J}=\{1,4,5,9,10\}$

the elements of the diagonal matrices $\mathbf{D}_{u}$ take only 2 values, $\sigma^{2}$ and $\sigma_{h}^{2} \triangleq \sigma^{2}+\sigma_{q}^{2}$ (which is the variance of noise and interference corresponding to the symbols that are hit)

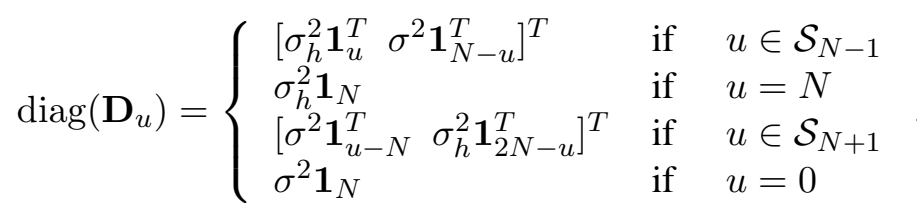

Further, denoting $\mathbf{C}_{u} \triangleq \mathbf{1 1 ^ { T }}+\mathbf{D}_{u}$, the pdf of the received signal is

$$
\begin{aligned}
f(\mathbf{x}) & =\sum_{u=0}^{2 N-1} p_{u} f(\mathbf{x} \mid U=u) \\
f(\mathbf{x} \mid U=u) & =\frac{1}{\pi^{N}\left|\mathbf{C}_{u}\right|} \exp \left(-\mathbf{x}^{H} \mathbf{C}_{u}^{-1} \mathbf{x}\right) .
\end{aligned}
$$

\section{The Bayesian Estimator}

The Bayesian MMSE estimator for the model considered is

$$
\hat{A}(\mathbf{x} ; \mathbf{p})=\mathbb{E}\{A \mid \mathbf{x}\}=\mathbf{1}^{T} \mathbb{E}_{U \mid \mathbf{x}}\left\{\mathbf{C}_{U}^{-1}\right\} \mathbf{x}
$$

Writing the expectation explicitly we have

$$
\hat{A}(\mathbf{x} ; \mathbf{p})=\mathbf{1}^{T}\left(\sum_{u=0}^{2 N-1} \frac{p_{u} f(\mathbf{x} \mid U=u)}{f(\mathbf{x})} \mathbf{C}_{u}^{-1}\right) \mathbf{x} .
$$

The performance of the Bayesian MMSE estimator is given by the MMSE

$$
\mathcal{E}(\mathbf{p})=\mathbb{E}\left[|A|^{2}\right]-\mathbb{E}\left[|\hat{A}|^{2}\right] .
$$

The MMSE estimator and its MMSE are function of the distribution of the random variable $U$, i.e., the vector $\mathbf{p}$. Since the MMSE estimator and its MSE are nonlinear functions of $\mathbf{p}$, we consider the following lower bound.

\section{The Genie Lower Bound on the MMSE}

A lower bound on the MMSE is given by the performance of a receiver helped by a genie who provides the current value of $U$, i.e., the position of the interference packet with respect to the training symbols. In this case, for each value $U=u$ we have a Gaussian model, for which the MMSE and its MSE are well known. The genie estimator assumes the random variable $U$ known:

$$
\tilde{A}(\mathbf{x}, U) \triangleq \mathbb{E}\{A \mid \mathbf{x}, U\}=\mathbf{1}^{T} \mathbf{C}_{U}^{-1} \mathbf{x} .
$$

Its MSE for each $U=u$ is given by $m(u)$ and the averaged MSE by $\xi(\mathbf{p})$

$$
\begin{aligned}
m(u) & \triangleq \mathbb{E}\left\{|\tilde{A}(\mathbf{x}, U)-A|^{2} \mid U=u\right\}=1-\mathbf{1}^{T} \mathbf{C}_{u}^{-1} \mathbf{1} \\
\xi(\mathbf{p}) & \triangleq \mathbb{E}\left\{|\tilde{A}(\mathbf{x}, U)-A|^{2}\right\} \\
& =\sum_{u=0}^{2 N-1} p_{u} \mathbb{E}\left\{|\tilde{A}(\mathbf{x}, U)-A|^{2} \mid U=u\right\} .
\end{aligned}
$$

The MSE $\xi(\mathbf{p})$ is called the genie lower bound and it satisfies

$$
\xi(\mathbf{p}) \leq \mathbb{E}\left\{|\hat{A}(\mathbf{x} ; \mathbf{p})-A|^{2}\right\}
$$

The genie lower bound can be optimized with respect to $\mathbf{p}$. The result is given below.

Theorem 1 The genie lower bound $\xi(\mathbf{p})$ is minimized if the training symbols are placed in two equal or quasi-equal clusters at the two ends of the data packet. Formally, denoting

$\bar{p}_{u}=\left\{\begin{array}{cll}\varepsilon & \text { if } & u \notin\left\{\left\lfloor\frac{N}{2}\right\rfloor, N+\left\lfloor\frac{N}{2}\right\rfloor, 0\right\} \\ (B-N+1) \varepsilon & \text { if } & u \in\left\{\left\lfloor\frac{N}{2}\right\rfloor, N+\left\lfloor\frac{N}{2}\right\rfloor\right\} \\ 0 & \text { if } & u=0\end{array}\right.$,

we have

$$
\left\{\overline{\mathbf{p}}, \overline{\mathbf{p}}^{\leftarrow}\right\}=\underset{\mathbf{p} \in \mathcal{P}}{\arg \min }\{\xi(\mathbf{p})\} .
$$

Note that $\overline{\mathbf{p}}=\overline{\mathbf{p}}^{\leftarrow}$ if $N$ is even.

The following theorem and corollary [6] characterize the asymptotic behavior of the genie bound and MMSE.

Theorem $\mathbf{2}$ Let $\xi\left(\mathbf{p} ; \sigma_{q}^{2}\right)$ and $\mathcal{E}\left(\mathbf{p} ; \sigma_{q}^{2}\right)$ be the genie bound and the MMSE respectively, where the dependence on the power of the interference $\sigma_{q}^{2}$ has been shown explicitly. For any choice of $\left(B, N, \sigma^{2}\right)$ we have

$$
\lim _{\sigma_{q}^{2} \rightarrow \infty}\left(\mathcal{E}\left(\mathbf{p} ; \sigma_{q}^{2}\right)-\xi\left(\mathbf{p} ; \sigma_{q}^{2}\right)\right)=0 .
$$

Corollary 1 For any choice of the system parameters $(B$, $\left.N, \sigma^{2}\right)$, there is a level of interference $\bar{\sigma}_{q}^{2}\left(B, N, \sigma^{2}\right)$ such that the placements $\overline{\mathbf{p}}$ and $\overline{\mathbf{p}} \leftarrow$ are the only placements that minimize the MMSE (8) for all $\sigma_{q}^{2}>\bar{\sigma}_{q}^{2}\left(B, N, \sigma^{2}\right)$. 


\section{RELIABLE DATA TRANSMISSION RATES UNDER ASYNCHRONOUS INTERFERENCE}

In this section we investigate the influence of the placement of training symbols on the data transmission performance. In addition to the setup in Section 2, we consider that the transmitter uses BPSK signaling with coding and interleaving. The coding and interleaving is done to decompose the communication channel into $B-N$ parallel independent channels, one channel for each of the data symbols within a packet. More exactly, the data stream is partitioned into $B-N$ substreams which are then coded independently. The binary symbols of the codewords of one substream are inserted in the same $k$-th position in successive data packets.

For each data packet the receiver first obtains a channel estimate using the training symbols. After the channel estimates for successive data packets are obtained, the substreams are decoded independently. As mentioned in the introduction, to make the model tractable, we assume that the receiver uses the genie estimator instead of the true MMSE estimator.

The performance measure considered is the achievable data transmission rate using the mutual information between the input and the output. Although the use of genie estimator will produce upper bounds to the achievable rate by the system considered, if the power of the interference is high, the use of genie and MMSE estimates provides identical results.

Two decoders are considered - a decoder that does hard detection of the binary symbols and then decoding and a decoder that uses the received data symbols and the channel estimates without intermediate processing.

\section{Reliable Rate with Hard Reception}

The asynchronous interference makes different data symbols be received under very different SINR levels. If the power of the interference is high, the data symbols that are hit can't be recovered (the probability of detection error approaches one half). Besides this, if all the training symbols are hit by the interference, no data symbol of that packet can be recovered reliably, regardless it is hit by the interference or not. However, since the interference power is high, these two situations can be detected accurately by the receiver and declared erasure events. Thus the hard detection performed by the receiver is ternary; after detection each data channel is equivalent to a binary channel with erasures.

From the transmission model (1), the $k$-th received symbol (assume $k \notin \mathcal{J}$,i.e., data symbol) is given by

$$
y_{k}=s_{k} A+z_{k} .
$$

Introduce the binary random variable $H_{k} ;\left\{H_{k}=1\right\}$ is the event that the $k$-th received symbol is hit by interference and $\left\{H_{k}=0\right\}$ its complement. Conditioned on $H_{k}, z_{k}$ is Gaussian; $z_{k} \mid H_{k} \sim \mathcal{C N}\left(0, \sigma^{2}+H_{k} \sigma_{q}^{2}\right)$. When the power of the interference is high, the erasure events can be detected accurately, i.e., besides $U$, the detector knows $H_{k}$. Assuming equiprobable input symbols, the ML detector is

$$
\tilde{s}_{k} \triangleq\left\{\begin{array}{lll}
\text { erasure } & \text { if } \quad \begin{array}{l}
H_{k}=1 \text { or } U=N \\
\text { otherwise }
\end{array} .
\end{array}\right.
$$

If the decoder does not use the information provided by the data transmission part anymore, the achievable rate for channel $k$ is given by the capacity of the binary channel with erasures

$\underline{C}_{k}=\left(1-P_{k, r}\right)\left(1+P_{k, c} \log _{2}\left(P_{k, c}\right)+\left(1-P_{k, c}\right) \log _{2}\left(1-P_{k, c}\right)\right)$,

where the probability of erasure $P_{k, r}$ and the probability of error (crossover) $P_{k, c}$ are respectively given by

$$
\begin{aligned}
P_{k, r} & =\mathbb{P}\left\{\left\{H_{k}=1\right\} \cup\{U=N\}\right\} \\
& =\left\{\begin{array}{lll}
\frac{B+\left(t_{1}-k\right)}{2 B-1} & \text { if } t_{1}>1 \text { and } 1 \leq k<t_{1} \\
\frac{B+\left(k-t_{N}\right)}{2 B-1} & \text { if } & t_{N}<B \text { and } t_{N}<k \leq B \\
\frac{B}{2 B-1} & \text { otherwise }
\end{array}\right. \\
P_{k, c} & =\mathbb{P}\left\{\tilde{s}_{k} \neq s_{k} \mid\left(\left\{H_{k}=1\right\} \cup\{U=N\}\right)^{C}\right\} .
\end{aligned}
$$

The probabilities $P_{k, c}$ can be obtained function of the probabilities $\mathbb{P}\left\{\tilde{s}_{k} \neq s_{k} \mid U, H_{k}=0\right\}$ and the placement. Conditioned on $H_{k}$ and $U$ the received signal $y_{k}$ is Gaussian, thus $\mathbb{P}\left\{\tilde{s}_{k} \neq s_{k} \mid U, H_{k}=0\right\}$ can be computed using the well-known formula for the probability of error of BPSK signaling in Rayleigh fading [7]. The result is

$$
\begin{aligned}
\theta(u, h) & \triangleq \mathbb{P}\left\{\tilde{s}_{k} \neq s_{k} \mid U=u, H_{k}=0\right\} \\
& =\frac{1}{2}\left(1-\sqrt{\frac{\mathbf{1}^{T} \mathbf{C}_{u}^{-1} \mathbf{1}}{\sigma^{2}+1}}\right) .
\end{aligned}
$$

The achievable rate of the system (lower bound on capacity) of the system considered is given by the average

$$
\underline{C}=\frac{1}{B} \sum_{k \notin \mathcal{J}} \underline{C}_{k} .
$$

The placement that optimizes this lower bound cannot be found in closed form. Moreover, the numerical evaluations indicate that the problem has different solutions for different choices of the parameters $\left(B, N, \sigma^{2}\right)$. Note that if the noise has moderately low variance, at high interference power, the variation of the probabilities of error $\mathbb{P}\left\{\tilde{s}_{k} \neq s_{k} \mid H_{k}=\right.$ $0, U\}$ with $u$ is small and the placement influence on the 
achievable rate is mainly through the probabilities of erasure $P_{k, r}$. For example, if the training symbols are placed in one cluster, then the data symbols from the first and last data clusters have high probability of erasure. This can lower significantly the performance of the system, as shown and discussed further in the numerical results section 5 .

\section{Reliable Rate with Soft Reception}

The previous computation is valid only for high values of the interference power. A performance measure valid for all values of $\sigma_{q}^{2}$ can be obtained considering a decoder that processes the soft outputs coherently using the genie estimator, by computing numerically the mutual information between the input and the output $I\left(s_{k} ; y_{k}, U, \tilde{A}(\mathbf{x}, U)\right)$. Because the genie estimator is used, this measure is an upper bound on the achievable rate of the system under the transmission and decoding constraints considered. The upper bound on the achievable rate of the transmission system under the constraints imposed is the average of the rates of the $B-N$ parallel independent channels.

Denote by $q_{0}$ the distribution of the data symbols $s_{k}$. Using the independence between $s_{k}$ and the random variables $U$ and $\tilde{A}(\mathbf{x}, U)$ as well as the symmetry of the model, the upper bound on the capacity of channel $k$ can be obtained as

$$
\begin{aligned}
\bar{C}_{k} & =\max _{q_{0}}\left\{I\left(s_{k} ; y_{k}, U, \tilde{A}(\mathbf{x}, U)\right)\right\} \\
& =\mathbb{E}\left\{\left.I\left(q_{0}\left(s_{k}\right) ; f\left(y_{k} \mid s_{k}, U, \tilde{A}(\mathbf{x}, U)\right)\right)\right|_{q_{0}=\left\{\frac{1}{2}, \frac{1}{2}\right\}}\right\} .
\end{aligned}
$$

The distribution $f\left(y_{k} \mid s_{k}, U, \tilde{A}(\mathbf{x}, U)\right)$ is a mixture of two complex Gaussian distributions with variances $\sigma_{1}^{2}(U) \triangleq$ $1-\mathbf{1}^{T} \mathbf{C}_{U}^{-1} \mathbf{1}+\sigma^{2}$ and $\sigma_{2}^{2}(U) \triangleq 1-\mathbf{1}^{T} \mathbf{C}_{U}^{-1} \mathbf{1}+\sigma^{2}+\sigma_{q}^{2}$ :

$$
\begin{aligned}
f\left(y_{k} \mid s_{k}, U, \tilde{A}\right)= & \mathbb{P}\left\{H_{k}=0 \mid U\right\} \mathcal{C N}\left(y_{k} ; s_{k} \tilde{A}, \sigma_{1}^{2}(U)\right) \\
& +\mathbb{P}\left\{H_{k}=1 \mid U\right\} \mathcal{C N}\left(y_{k} ; s_{k} \tilde{A}, \sigma_{2}^{2}(U)\right)
\end{aligned}
$$

So the upper bound on the capacity of channel $k$ is obtained by averaging the capacities of the channels given by each $U$ and $\tilde{A}(\mathbf{x}, U)$.

The upper bound on the achievable rate is

$$
\bar{C}=\frac{1}{B} \sum_{k \notin \mathcal{J}} \bar{C}_{k} .
$$

Because of the mixture of distributions, this upper bound can be calculated only numerically; results and further comments are provided in the next section.

\section{NUMERICAL RESULTS}

The simulations were done for the following parameters: $B=80, N=6, \sigma^{2}=-16.9 d B, \sigma_{A}^{2}=1$.

First, we plot the performance of the MSE of the channel estimator and the genie bound for the three schemes represented in Fig. 2. For the "optimal" and the "middle"

(a) 2 clusters of equal size, "optimal"

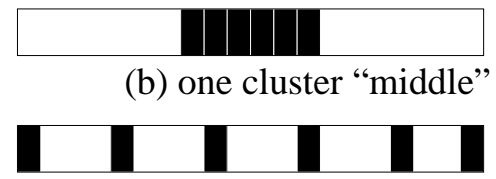

(c) "spread"

Fig. 2: The training schemes compared in Fig. 3

placements, the genie bounds are relatively tight for all $\sigma_{q}^{2}$. The interesting fact is that the MMSE of the "spread" placement scheme has a bell shape and the genie bound in not tight. This can be explained by thinking that the coefficients $f(U=u \mid \mathbf{x})$ in the expression (7) of the MMSE estimator act like an embedded maximum a posteriori (MAP) soft detector. The detection can be done better if there are fewer events with high a priori probabilities. This happens if the symbols are grouped into two clusters placed at the edges or in one cluster; in these cases the MMSE is close to the genie bound. The previous argument works very well when the interference power has moderately high values; at high values of the interference power the detection can be done accurately for any placement.

Numerical results for the lower bound $\underline{C}$ given by (15)

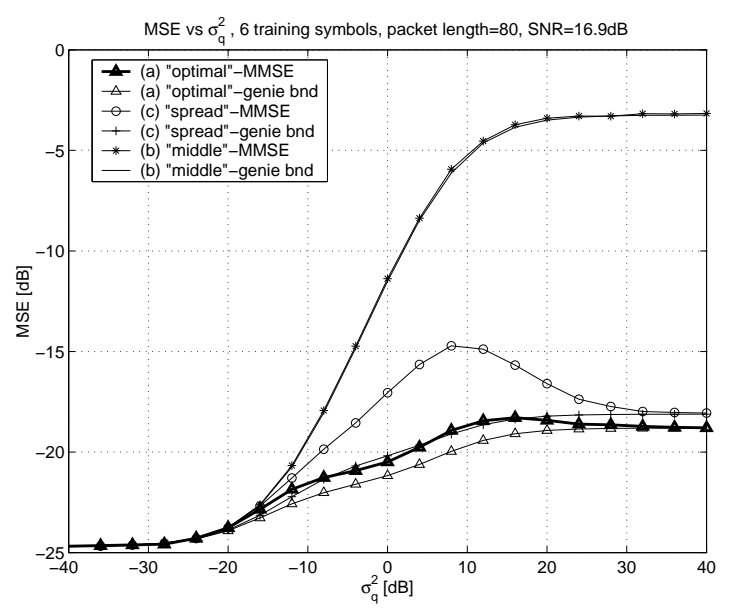

Fig. 3: The performance of the "optimal", "middle" and "spread" placements and their genie bounds

on the achievable rate of the binary channel with errors and erasures when $\sigma_{q}^{2} \rightarrow \infty$ are given in Table 1. First, it can be observed that the achievable rate can be almost doubled by 


\begin{tabular}{||l|l||}
\hline \hline Placement $-\mathcal{J}$ & $\underline{C}[$ bps $]$ \\
\hline$\overline{\mathcal{J}}=\{1,2,3,78,79,80\}$ & 0.4329 \\
\hline $\mathcal{J}_{\text {middle }}=\{38, \ldots, 43\}$ & 0.3313 \\
\hline $\mathcal{J}_{\text {one edge }}=\{1, \ldots, 6\}$ & 0.2282 \\
\hline $\mathcal{J}_{\text {max }}=\{1,9,28,53,72,80\}$ & 0.4334 \\
\hline \hline
\end{tabular}

Table 1: The lower bound on the achievable rate of the binary channel with errors and erasures, $\left(B=80, N=6, \sigma^{2}=-16.9 d B, \sigma_{A}^{2}=1\right.$, $\left.\sigma_{q}^{2} \rightarrow \infty\right)$

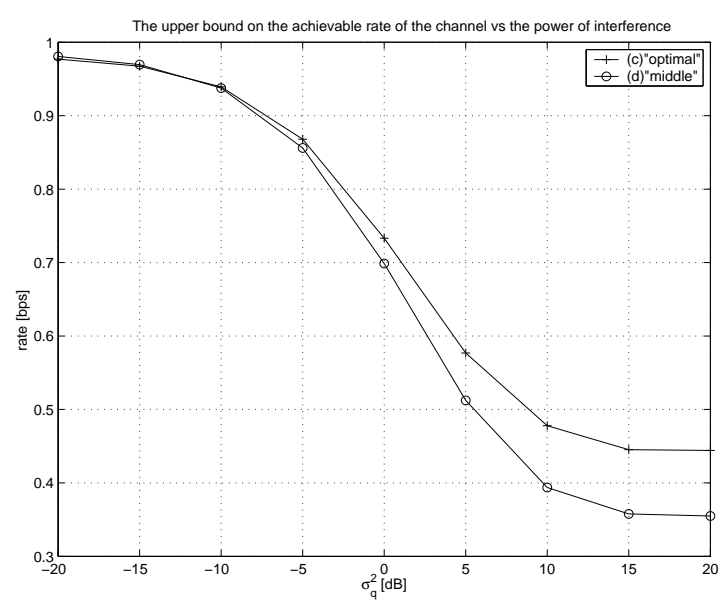

Fig. 4: The upper bound on the achievable rate of the communication system of Section $4 ; B=80$, $N=6, \sigma^{2}=-16.9 d B, \sigma_{A}^{2}=1$.

selecting the right placement. The big difference is given by the erasures that occur when all training symbols are hit. However, once the packet begins and ends with a training symbol, the influence of the training placement is relatively low. The placement $\mathcal{J}_{\max }$ that maximizes the bound was found by numerical evaluations. Except that it is symmetric, this placement does not correspond to a regular scheme (e.g.,is not uniform). Moreover, if the system parameters are changed, the placement that maximizes $\underline{C}$ is different; however, the observations made before still hold.

In Fig. 4 is represented the upper bound on the achievable rate (17) for the "optimal" and "middle" placements.

It can be seen that at high values of the interference power the improvement is around $0.1 \mathrm{bps}$, or $30 \%$. The numerical values obtained for high $\sigma_{q}^{2}$ are very close to those given in Table 1; in this situation (low noise power, high interference power) the loss induced by considering the channel as a binary channel with erasures is negligible.

\section{CONCLUSIONS}

For a packet data transmission system under asynchronous interference, we considered the effect of training symbols placement on the achievable data rates. The receiver obtains first an estimate of the channel using the training symbols and then decodes the data coherently. For the achievable data rates under the constraints given, we obtain an upper and a lower bound. These bounds can be optimized only numerically; the solution depends on the parameters of the system. The numerical results show that by using the placement optimized for the channel estimation performance the data rate loss is negligible; thus the effect of the placement on the data rate is mainly through the efficiency of channel estimation. Also, it is shown that the achievable rates can be doubled by selecting the right placement scheme.

\section{REFERENCES}

[1] R. Negi and J. Cioffi, "Pilot tone selection for channel estimation in a mobile OFDM System," IEEE Trans. on Consumer Electronics, vol. 44, pp. 1122-1128, Aug. 1998.

[2] S. Adireddy, L. Tong, and H. Viswanathan, "Optimal placement of known symbols for frequency-selective block-fading channels," IEEE Trans. Info. Theory, vol. 48, pp. 2338-2353, August 2002.

[3] M. Dong and L. Tong, "Optimal Design and Placement of Pilot Symbols for Channel Estimation," IEEE Trans. on Signal Processing, vol. 50, pp. 3055-3069, December 2002.

[4] S. Ohno and G. B. Giannakis, "Optimal Training and Redundant Precoding for Block Transmissions with Application to Wireless OFDM," IEEE Trans. on Communications, vol. 50, December 2002.

[5] C. Budianu and L. Tong, "Channel Estimation for SpaceTime Block Coding Systems," IEEE Trans. Signal Processing, vol. 50, pp. 2515-2528, Oct 2002.

[6] C. Budianu and L. Tong, "Channel Estimation under Asynchronous Packet Interference," submitted to IEEE Trans. on Signal Processing, Apr. 2003. http://acsp.ece.cornell.edu/pubJ.html.

[7] J. Proakis, Digital Communications. McGraw Hill, 4 ed., 2001. 